Homology, Homotopy and Applications, vol.6(1), 2004, pp.413-437

\title{
INEQUILOGICAL SPACES, DIRECTED HOMOLOGY AND NONCOMMUTATIVE GEOMETRY
}

\author{
MARCO GRANDIS \\ (communicated by George Janelidze)
}

\begin{abstract}
We introduce a preordered version of D. Scott's equilogical spaces [29], called inequilogical spaces, as a possible setting for Directed Algebraic Topology. The new structure can also express 'formal quotients' of spaces, which are not topological spaces and are of interest in noncommutative geometry, with finer results than the ones obtained with equilogical spaces, in a previous paper.

This setting is compared with other structures which have been recently used for Directed Algebraic Topology: spaces equipped with an order, or a local order, or distinguished paths, or distinguished cubes.
\end{abstract}

\section{Introduction}

This work is devoted to the interaction between two recent subjects: Scott's equilogical spaces and Directed Algebraic Topology. It is a sequel of a previous one, cited as Part I [17], where we showed how equilogical spaces are able to express 'formal quotients' of interest in noncommutative geometry ('noncommutative tori'), which can be explored extending singular homology. Here, we introduce a directed (preordered) version of such a structure, called inequilogical space, which can be explored by preordered homology groups and gives finer results in expressing those 'formal quotients'.

An equilogical space $X=\left(X^{\sharp}, \sim\right)[\mathbf{2 9}]$ is a topological space $X^{\sharp}$ equipped with an equivalence relation $\sim$; a map of equilogical spaces $X \rightarrow Y$ is a mapping $X^{\sharp} / \sim$ $\rightarrow Y^{\sharp} / \sim$ which admits some continuous lifting $X^{\sharp} \rightarrow Y^{\sharp}$. Note that we drop the usual condition that $X^{\sharp}$ be $T_{0}$ (I.1.2.); therefore, the category Eql thus obtained contains Top as a full subcategory, identifying a space $T$ with the pair $\left(T,=_{T}\right) ; \mathbf{E q l}$ has 'finer' quotients and is Cartesian closed. In Part I we have seen that singular homology can be extended to equilogical spaces, with similar properties, and can give interesting results even when the underlying space $|X|=X^{\sharp} / \sim$ is trivial.

On the other hand, Directed Algebraic Topology is a recent subject, whose present applications deal mainly with concurrency. Its domain should be distin-

Received January 20, 2004, revised September 6, 2004; published on October 18, 2004. 2000 Mathematics Subject Classification: 18B30, 54A05, 55U10, 55Nxx, 46L80.

Key words and phrases: Equilogical spaces, cubical sets, singular homology, directed homology, noncommutative $\mathrm{C}^{*}$-algebras.

(C) 2004, Marco Grandis. Permission to copy for private use granted. 
guished from classical Algebraic Topology by the principle that directed spaces have privileged directions and their paths need not be reversible. Its homotopical and homological tools are similarly 'non-reversible': directed homotopies, fundamental categories, directed homology. Its applications can deal with domains where privileged directions appear, like concurrent processes, traffic networks, space-time models, etc. [14].

As a topological setting to develop this subject, various structures combining topology and order have been considered in the theory of concurrency $[\mathbf{9}, \mathbf{1 0}, \mathbf{1 1}$, 24]. However, for developing a general theory of Directed Algebraic Topology, such notions present various drawbacks (1.3): the lack of essential models or the lack of cones and suspension. These problems can be overcome with more complex structures, like spaces with distinguished paths $[\mathbf{1 2}, \mathbf{1 3}]$, cubical sets and spaces with distinguished cubes $[\mathbf{1 5}, \mathbf{1 6}]$. Moreover, such structures also contain models of 'formal spaces' of interest in noncommutative geometry, which cannot be realised as topological spaces.

Developing a remark in $[\mathbf{1 5}], 6.4$, we introduce and study here a simpler setting which can still express those 'formal quotients'. An inequilogical space $X=\left(X^{\sharp}, \sim\right)$ is defined as a preordered topological space $X^{\sharp}$ equipped with an equivalence relation; a morphism is defined as above, requiring a continuous preorder-preserving lifting. The category pEql so obtained is studied in Section 1. Inequilogical spaces have singular cubes defined on the standard ordered cubes $\uparrow \mathbf{I}^{n}$ and a directed homology consisting of preordered abelian groups $\uparrow H_{n}(X)$ (Section 3). To understand how easily and effectively this new category can express 'privileged directions' and give rise to directed paths, it suffices to consider the following model of the 'directed circle', the inequilogical space $(\uparrow \mathbf{R}, \equiv \mathbf{z})$, i.e. the quotient (in $\mathbf{p E q l})$ of the ordered line $\uparrow \mathbf{R}$ modulo the action of the subgroup $\mathbf{Z}$.

Section 4 deals with formal quotients of preordered spaces as inequilogical spaces, treating in detail one example. The subgroup $G_{\vartheta}=\mathbf{Z}+\vartheta \mathbf{Z} \subset \mathbf{R}(\vartheta$ irrational $)$ acts on the line by translations; being dense in the line, it has a coarse orbit space $\mathbf{R} / G_{\vartheta}$. Replacing this trivial space with the quotient cubical set $C_{\vartheta}=(\square \uparrow \mathbf{R}) / G_{\vartheta}([\mathbf{1 5}]$, $4.2 \mathrm{~b}$ ) derived from the order-preserving cubes $\mathbf{I}^{n} \rightarrow \mathbf{R}$, or equivalently with the inequilogical space $C_{\vartheta}^{\prime}=\left(\uparrow \mathbf{R}, \equiv_{G_{\vartheta}}\right)$, we have a non-trivial object, whose directed homology

$$
\uparrow H_{1}\left(\uparrow \mathbf{R}, \equiv_{G_{\vartheta}}\right)=\uparrow H_{1}\left((\square \uparrow \mathbf{R}) / G_{\vartheta}\right) \cong \uparrow G_{\vartheta},
$$

is able to recover the totally ordered group $\uparrow G_{\vartheta} \subset \uparrow \mathbf{R}$ (up to isomorphism) and the irrational number $\vartheta$, up to the corresponding equivalence relation (Thm. 4.4).

All this agrees with the irrational rotation $\mathrm{C}^{*}$-algebra $A_{\vartheta}$, which 'replaces' in noncommutative geometry the trivial quotient $\mathbf{R} / G_{\vartheta}$ and the trivial leaf space of the corresponding Kronecker foliation on the torus $[\mathbf{5 , 6 , 2 3 , 2 5 ] . ~ T h e ~ p r e s e n t ~}$ models, however, seem to be geometrically more evident than the corresponding $\mathrm{C}^{*}$-algebras; direction plays a recognisable role, since the homology groups of the equilogical space $\left(\mathbf{R}, \equiv_{G_{\vartheta}}\right)$ do not allow to reconstruct $\vartheta$, at any extent (see Part I).

The classification of the inequilogical spaces $C_{\vartheta}^{\prime}=\left(\uparrow \mathbf{R}, \equiv_{G_{\vartheta}}\right)$ is extended in Section 5, taking $G_{\vartheta}=\sum_{i} \vartheta_{i} \mathbf{Z}$ where the numbers $\left(\vartheta_{1}, \ldots, \vartheta_{n}\right)$ are linearly independent on $\mathbf{Q}$. Finally, various inequilogical structures of the $n$-torus are studied in 
Section 6, determining their directed homology.

Equilogical spaces have been introduced in $[\mathbf{2 9}]$; see also $[\mathbf{1}, \mathbf{2}, \mathbf{2 7}, \mathbf{2 8}]$. References and motivation for Directed Algebraic Topology can be found in [12]; for cubical sets in $[\mathbf{1 5}]$. Within category theory, $\mathrm{pEq}$ can be viewed as the regular completion $\mathrm{pTop}_{\text {reg }}$ of the category of preordered spaces [4]. One can use this fact to prove that pEql is Cartesian closed, as in $[\mathbf{2 8}]$ (p. 161) for $\mathbf{E q l}=\mathbf{T o p}_{\text {reg }}$; but here we rather need an explicit construction of some internal homs (1.8).

A preorder is a reflexive and transitive relation; an order is also antisymmetric. Structures provided with some sort of direction are usually distinguished by the prefix $\uparrow$. A map between spaces is a continuous mapping. The index $\alpha$ always takes values 0,1 . The reference I.1 applies to Section 1 of Part I [17]; similarly I.1.2 or I.1.2.3 refer to its Subsection 1.2 or item (3) of the latter.

\section{Inequilogical spaces and directed topology}

Inequilogical spaces can be seen as 'formal quotients' of preordered topological spaces, and used as a simple setting for Directed Algebraic Topology.

\subsection{Equilogical spaces}

Let us recall that an equilogical space $X=\left(X^{\sharp}, \sim\right)$ is a topological space $X^{\sharp}$ (the support) provided with an equivalence relation, written $\sim_{X}$ or $\sim$; the underlying space (or set, when convenient) is the quotient $|X|=X^{\sharp} / \sim$. A map of equilogical spaces $f: X \rightarrow Y$ is a mapping $f:|X| \rightarrow|Y|$ which admits some continuous lifting $f^{\prime}: X \rightarrow Y$; or, equivalently, an equivalence class of continuous mappings $f^{\prime}: X \rightarrow$ $Y$ respecting the equivalence relations

$$
\forall x, x^{\prime} \in X: \quad x \sim_{X} x^{\prime} . \Rightarrow . f^{\prime}(x) \sim_{Y} f^{\prime}\left(x^{\prime}\right),
$$

under the associated pointwise equivalence relation

$$
f^{\prime} \sim f^{\prime \prime} \text { if }\left(\forall x \in X: f^{\prime}(x) \sim_{Y} f^{\prime \prime}(x)\right) .
$$

The category Eql thus obtained contains Top as a full subcategory, identifying the space $X$ with the obvious pair $\left(X,=_{X}\right)$. (Equilogical spaces have been introduced in $[\mathbf{2 9}]$ using $T_{0}$-spaces as supports, so that they can be viewed as subspaces of algebraic lattices with the Scott topology, which is always $T_{0}$. Their category, a full subcategory of Eql, is often written as Equ.)

An equilogical space $X$ is isomorphic to a topological space $A$ if and only if $A$ is a retract of $X^{\sharp}$, with a retraction $p: X^{\sharp} \rightarrow A$ whose equivalence relation is precisely $\sim_{X}$. But the new category has relevant new objects (cf. 1.4).

In Part I we have extended singular homology to equilogical spaces, to study objects of which we have no direct geometric intuition. As in Massey's text [22], we have followed the cubical approach instead of the more usual simplicial one. General motivations for preferring cubes essentially go back to the fact that cubes are closed under product, while tetrahedra are not. But here, a specific, strong motivation will be our use of the natural order on the standard cube $\mathbf{I}^{n}=[0,1]^{n}$ to define directed homology of inequilogical spaces (cf. 3.2). 


\subsection{Privileged directions}

As recalled in the Introduction, Directed Algebraic Topology is concerned with 'directed spaces', having privileged directions and directed paths, generally nonreversible. Its applications, mostly developed within the theory of concurrency, can also deal with the analysis of space-time models, 'directed images', traffic networks, etc. (cf. $[\mathbf{1 2}, \mathbf{1 4}]$ and references there); but here we shall restrict our attention to theoretical and internal aspects.

It is not obvious how one can modify or enrich topological spaces, to produce a 'good' structure with such features. Clearly, we are not looking for orientation, which - to begin with - is unable to give privileged paths in dimension greater than 1; moreover, non orientable manifolds can have non-trivial directed structures (1.7).

The pictures below show four situations we want to be able to formalise, within the square $Q=[0,5] \times[0,5]$ of the euclidean plane

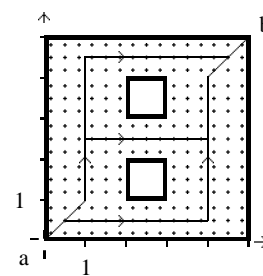

$X$

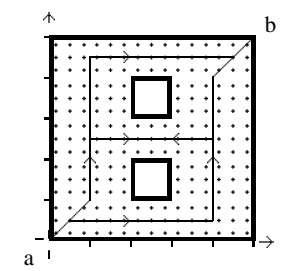

Y

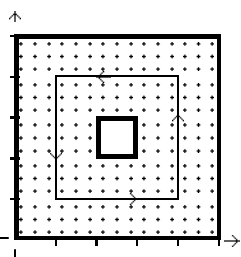

Z

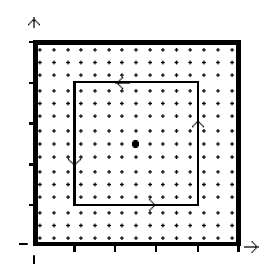

$W$

(a) First, let us consider the compact subspace $X=Q \backslash(] 2,3[\times(] 1,2[\cup] 3,4[))$ as an ordered topological space, with the natural (partial) order of the plane: $(s, t) \leqslant$ $\left(s^{\prime}, t^{\prime}\right)$ if and only if $s \leqslant s^{\prime}$ and $t \leqslant t^{\prime}$. Thus, defining a directed path as any orderpreserving map $\uparrow \mathbf{I} \rightarrow X$ on the standard ordered interval $\uparrow \mathbf{I}=\uparrow[0,1]$, there are essentially three paths from the minimum $a=(0,0)$ to the maximum $b=(5,5)$, up to (the equivalence relation generated by) directed homotopy of directed paths (parametrised on $\uparrow \mathbf{I} \times \uparrow \mathbf{I}$, with fixed endpoints).

(b) Second, let us consider $Y$ as the same space with the preorder relation $(s, t) \prec$ $\left(s^{\prime}, t^{\prime}\right)$ defined by $t \leqslant t^{\prime}$. Now, directed paths have to move 'weakly upwards' but are free of wandering from right to left or vice versa; there are thus four homotopy classes of directed paths, from $a$ to $b$.

(c) Finally, we want that directed paths in $Z$ and $W$ turn around the centre, counterclockwise - being free of wandering with respect to their distance from the centre (the underlying spaces of these 'structures' are $Q \backslash] 2,3\left[{ }^{2}\right.$ and $Q$, respectively).

Plainly, the last two cases cannot be expressed by a preorder, but require a richer setting (for instance, they will be realised as inequilogical spaces, in 1.7). Case (b) shows that it is not convenient to restrict to order relations.

\subsection{Preordered spaces}

As we have seen, the category pTop of preordered topological spaces (spaces with a preorder relation, under no condition) and preorder-preserving maps already contains some models we are interested in. 
As standard objects of interest, let us consider: the ordered line $\uparrow \mathbf{R}$ (with natural order); the $n$-dimensional real ordered space $\uparrow \mathbf{R}^{n}$, with the product order $(x \leqslant y$ if $\left.x_{i} \leqslant y_{i}, \forall i\right)$; the standard ordered interval $\uparrow \mathbf{I}=\uparrow[0,1] \subset \uparrow \mathbf{R}$ and the standard ordered cube $\uparrow \mathbf{I}^{n} \subset \uparrow \mathbf{R}^{n}$.

A directed path in a preordered space $X$ is obviously defined as a morphism $\uparrow \mathbf{I} \rightarrow X$. This shows that it is convenient to identify a topological space $X$ with the chaotic-preordered space $\left(X, \approx_{X}\right)$, so that all (continuous) paths $\mathbf{I} \rightarrow X$ are still admissible morphisms $\uparrow \mathbf{I} \rightarrow\left(X, \approx_{X}\right)$. Thus, $\mathbf{R}^{n}$ will have the chaotic preorder and $\mathbf{R} \times \uparrow \mathbf{R}$ a product preorder, chaotic in the first variable and natural in the second. The spaces $X, Y$ considered in (4) can be viewed in pTop, as subobjects of $\uparrow \mathbf{R}^{2}$ or $\mathbf{R} \times \uparrow \mathbf{R}$, respectively.

In itself, pTop has rather good categorical properties (all limits and colimits exist; the ordered interval is exponentiable). But it cannot express models we would like to have, as a 'directed circle' or the two last examples above (in (4)).

One could extend pTop by some local notion of ordering - as in the usual geometric models of concurrent processes. The simplest way is perhaps to consider spaces equipped with a relation $\prec$ which is reflexive and locally transitive: every point has some neighbourhood on which the relation is transitive (stronger properties have been used in the theory of concurrency). This yields a category lpTop $([\mathbf{1 2}], 1.4)$ which contains a model of the directed circle, as well as a model of the space $Z$ in (4). But a relevant internal drawback appears, which makes this setting inadequate for directed homotopy and homology: mapping cones and suspension are lacking. Indeed, a locally preordered space cannot have a 'pointlike vortex', as $W$ in (4) (where all neighbourhoods of the centre contain some non-reversible loop): whence it cannot realise the cone of the directed circle (as proved in detail in [12], 4.6 ).

\subsection{Inequilogical spaces}

A preordered version of equilogical spaces yields a very simple, partially satisfactory setting for Directed Algebraic Topology. The new category pEql is built on the category pTop, like equilogical spaces on Top.

An inequilogical space, or preordered equilogical space $X=\left(X^{\sharp}, \sim\right)$ will be a preordered topological space $X^{\sharp}$ endowed with an equivalence relation $\sim_{X}($ or $\sim)$; the preorder relation will generally be written as $\prec_{X}$. The quotient $|X|=X^{\sharp} / \sim$ will be viewed as a preordered topological space (with the induced preorder and topology), or a topological space, or a set, as convenient. A map $f: X \rightarrow Y$ 'is' a mapping $f:|X| \rightarrow|Y|$ which admits some continuous preorder-preserving lifting $f^{\prime}: X^{\sharp} \rightarrow Y^{\sharp}$. Equivalently, as in 1.1, a map is an equivalence class of maps $f^{\prime}$ in pTop which respect the equivalence relations (2), under the equivalence relation $f^{\prime} \sim f^{\prime \prime}$ (in (3)). Note that there are no mutual conditions among topology, preorder and equivalence relation.

This category will be denoted as pEql. The forgetful functor

$$
|-|: \text { pEql } \rightarrow \text { pTop, } \quad|X|=X^{\sharp} / \sim,
$$

with values in preordered topological spaces (or spaces, or sets, when convenient) has already been defined, implicitly; it sends the map $f: X \rightarrow Y$ to the underlying 
mapping $f:|X| \rightarrow|Y|$ (also written $|f|$ ). A point $x:\{*\} \rightarrow X$ is an element of the underlying space $|X|$.

Extending 1.3, the following embeddings will be viewed as inclusions (and again, the chaotic preorder on a set is written as $\approx$ )

$$
\begin{gathered}
\text { Top } \underset{J_{1} \downarrow}{\text { Eql } \underset{J_{4}}{\longrightarrow} \text { p pql }} \underset{\substack{J_{3} \\
\text { Top }}}{\longrightarrow} \\
J_{1}(T)=\left(T,=_{T}\right), \\
J_{3}(T, \prec)=\left(T, \prec,=_{T}\right), \quad J_{2}(T)=\left(T, \approx_{T}\right), \\
J_{4}(T, \sim)=\left(T, \approx_{T}, \sim\right) .
\end{gathered}
$$

Reversing the preorder relation gives the reflected, or opposite, inequilogical space

$$
(-)^{\mathrm{op}}: \mathrm{pEq} \mathbf{E} \rightarrow \mathrm{pEq} \mathbf{l}, \quad X^{\mathrm{op}}=\left(X^{\sharp}, \prec^{\mathrm{op}}, \sim_{X}\right) .
$$

The reflection $(-)^{\mathrm{op}}$ is a (covariant) involutive endofunctor. An object isomorphic to its reflection will be said to be reflexive, or self-dual; for instance, $\uparrow \mathbf{I}^{n}$ and $\uparrow \mathbf{R}^{n}$ are reflexive.

1.5 Theorem (Limits). The category $\mathrm{pEql}$ has all limits and colimits, constructed as in Eql and equipped with the appropriate preorder (as shown in detail in the proof).

Proof. The argument proceeds in the same way as the similar proof for equilogical spaces, in [1] or I.1.3, replacing Top with pTop; we write it down because we shall need the explicit construction of some limits and colimits. As well-known, it suffices to construct products, equalisers, sums (i.e., coproducts) and coequalisers.

A product $\prod X_{i}$ is the product of the preordered spaces $X_{i}^{\sharp}$, equipped with the product of all equivalence relations; a sum (or coproduct) $\sum X_{i}$ is the sum of the preordered spaces $X_{i}^{\sharp}$, with the sum of their equivalences.

Now, take two maps $f, g: X \rightarrow Y$. For their equaliser $E=\left(E^{\sharp}, \sim\right)$, take first the (set-theoretical) equaliser $E_{0}$ of the underlying mappings $f, g:|X| \rightarrow|Y|$; then the space $E^{\sharp}$ is the counterimage of $E_{0}$ in $X^{\sharp}$, with the restricted topology, preorder and equivalence relation; the map $E \rightarrow X$ is induced by the inclusion $E^{\sharp} \rightarrow X^{\sharp}$. For the coequaliser $C$ of the same maps, consider the set-theoretical coequaliser of the underlying mappings $f, g:|X| \rightarrow|Y|$, realised as a quotient $Y^{\sharp} / \sim_{C}$, modulo an equivalence relation containing $\sim_{Y}$. Then $C=\left(Y^{\sharp}, \sim_{C}\right)$, with the map $Y \rightarrow C$ induced by the identity of $Y^{\sharp}$ (and represented by the canonical projection $|Y| \rightarrow$ $|C|)$. Note that, as in Part I, coequalisers in Top (or pTop) are not used.

\subsection{Regular subobjects and quotients}

By definition, an inequilogical subspace of $X$ is any topological subspace of $X^{\sharp}$ saturated with respect to $\sim_{X}$, and equipped with the restricted structure. An inequilogical quotient of $X$ has the same support, with the same preorder and a coarser equivalence relation. (In fact, we have proved in 1.5 that any regular subobject 
$E \rightarrow X$ is an inequilogical subspace, as defined above; the converse is easily proved by taking the cokernel pair of $E \rightarrow X$; dually for quotients.)

To show how our new setting is more flexible and richer than pTop, it suffices to consider that the coequaliser in pTop of the faces of the ordered interval

$$
\partial^{0}, \partial^{1}:\{*\} \rightrightarrows \uparrow \mathbf{I}, \quad \partial^{0}(*)=0, \quad \partial^{1}(*)=1,
$$

is the circle $\mathbf{S}^{1}$ with the chaotic preorder (loosing any information of direction), while their coequaliser in $\mathbf{p E q} \mathbf{l}$ is produced by the equivalence relation $\mathbf{R}_{\partial \mathbf{I}}$ which identifies the endpoints

$$
\uparrow \mathbf{S}_{e}^{1}=\left(\uparrow \mathbf{I}, \mathbf{R}_{\partial I}\right)=\left(\mathbf{I}, \leqslant, \mathbf{R}_{\partial I}\right) \quad \text { (the standard inequilogical circle) }
$$

(as in Part I, $R_{A}$ will often denote the equivalence relation which collapses a subset A.)

It is important to note that this object still bears the natural order on the interval: thus, the directed paths $\uparrow \mathbf{I} \rightarrow \uparrow \mathbf{S}_{e}^{1}$ have to move in a precise direction, say 'counterclockwise' (moreover, local directed paths will be able to cross over the pasting point and turn around any number of times; cf. 2.3). Note also that, while in the non-directed case the distinction between the corresponding coequalisers, $\mathbf{S}^{1}$ and $\mathbf{S}_{e}^{1}$, is of a questionable interest (and, indeed, these objects are locally homotopy equivalent, cf. I.2.5), here the difference between the two coequalisers, $\mathbf{S}^{1}=\left(\mathbf{S}^{1}, \approx\right.$ $,=)$ and $\uparrow \mathbf{S}_{e}^{1}$, is essential.

\subsection{Other models}

Generalising the standard inequilogical circle (9), the standard n-dimensional inequilogical sphere $\uparrow \mathbf{S}_{e}^{n}$ will be defined as a quotient in $\mathrm{pEq}$ l of the ordered cube $\uparrow \mathbf{I}^{n}$, modulo the equivalence relation which identifies all points of the boundary

$$
\uparrow \mathbf{S}_{e}^{n}=\left(\uparrow \mathbf{I}^{n}, R_{\partial \mathbf{I}^{n}}\right)=\left(\mathbf{I}^{n}, \leqslant, R_{\partial \mathbf{I}^{n}}\right) \quad(n>0),
$$

while $\uparrow \mathbf{S}_{e}^{0}=(\{0,1\},=,=)$ has the discrete topology and order. All inequilogical spheres are reflexive. We shall see that all of them are pointed suspensions of $\uparrow \mathbf{S}_{e}^{0}$.

Also here $\uparrow \mathbf{S}_{e}^{1}$ is not isomorphic to the quotient of the ordered line modulo the action of $\mathbf{Z}$

$$
\uparrow \overline{\mathbf{S}}_{e}^{1}=\left(\uparrow \mathbf{R}, \equiv_{\mathbf{z}}\right)=\left(\mathbf{R}, \leqslant, \equiv_{\mathbf{Z}}\right) .
$$

In fact, directed paths in the object $\uparrow \overline{\mathbf{S}}_{e}^{1}$ can be concatenated, while in $\uparrow \mathbf{S}_{e}^{1}$ cannot, generally (see 2.1). Similarly, we have different higher spheres $\uparrow \overline{\mathbf{S}}_{e}^{n}=\left(\uparrow \mathbf{R}^{n}, \sim_{n}\right)$, where the equivalence relation $\sim_{n}$ is generated by the congruence modulo $\mathbf{Z}^{n}$ and by identifying all points $\left(t_{1}, \ldots, t_{n}\right)$ where at least one coordinate belongs to $\mathbf{Z}$.

Inequilogical models of the 'structures' $Z, W$ considered in (4) can be realised as subspaces of the counterclockwise inequilogical plane $H=\left(H^{\sharp}, \sim\right)$ : this is the preordered helicoid $H^{\sharp} \subset \mathbf{R} \times \mathbf{R} \times \uparrow \mathbf{R}$ described by the parametric equations $x=$ $\rho \cdot \cos (t), y=\rho \cdot \sin (t), z=t$ with the equivalence relation associated to the orthogonal projection on the $x y$-plane (and the preorder $z \leqslant z^{\prime}$ ). Note that $H$ also contains the circle $\uparrow \overline{\mathbf{S}}_{e}^{1}$, as the inequilogical subspace of points with $\rho=2 \pi$.

Various inequilogical structures of the torus will be studied in Section 6 . The Klein bottle (though a non-orientable manifold) can be given an inequilogical struc- 
ture locally isomorphic to $\uparrow \mathbf{I}^{2}$, namely the inequilogical quotient $\uparrow K=\left(\mathbf{I}^{2}, \leqslant, R_{K}\right)$ of a convenient ordered square $\left(\mathbf{I}^{2}, \leqslant\right)$ modulo the usual equivalence relation $R_{K}$ (described below 'on generators')

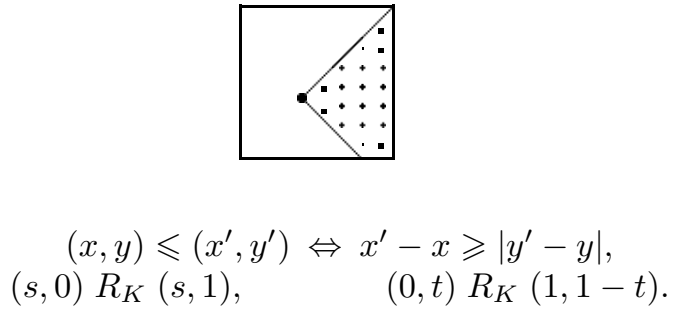

As recalled in the Introduction, pEql is Cartesian closed. Rather than giving a proof of this fact, by category-theoretical arguments, we give a direct construction of the internal homs $Y^{A}$ in a case largely covering the path-objects $Y^{\uparrow \mathbf{I}}$ we are interested in.

1.8 Theorem (Internal homs). Let $A$ be a preordered topological space, whose topology is Hausdorff, locally compact.

(a) A is exponentiable in pTop: for every preordered topological space $T$, the internal hom $T^{A}$ is the subspace of order-preserving maps $\operatorname{p} \operatorname{Top}(A, T) \subset \operatorname{Top}(A, T)$, with the (restricted) compact-open topology and the pointwise preorder

$$
h^{\prime} \prec_{E} h^{\prime \prime} \quad \text { if } \quad\left(\forall a \in A, h^{\prime}(a) \prec_{T} h^{\prime \prime}(a)\right) .
$$

(b) This construction can be extended to the inequilogical exponential $Y^{A}$, for $Y$ in pEql

$$
Y^{A}=\left(Y^{\sharp A}, \sim_{E}\right), \quad h^{\prime} \sim_{E} h^{\prime \prime} \text { if }\left(\forall a \in A, h^{\prime}(a) \sim_{Y} h^{\prime \prime}(a)\right),
$$

where $Y^{\sharp A}$ is the previous exponential, in $\mathrm{p}$ Top, and $\sim_{E}$ is the pointwise equivalence relation of maps $A \rightarrow Y^{\sharp}$ (cf. (3)).

(c) For every inequilogical space $X,|X \times A|=|X| \times A$.

(d) More generally, all this holds for every preordered topological space $A$ whose underlying space is exponentiable in Top, letting $T^{A}$ be the subspace of the topological exponential formed of the order-preserving maps, equipped with the pointwise preorder.

Proof. We only write down the proof of (a), since the rest is an easy adaptation of the proof of the analogous results for equilogical spaces (I.1.5).

Forgetting preorders, it is well-known that a Hausdorff, locally compact space $A$ is exponentiable in Top: $T^{A}$ is the space of maps $\operatorname{Top}(A, T)$ with the compactopen topology, and there is a natural bijection $\tau$, saying that the endofunctor $(-)^{A}:$ Top $\rightarrow$ Top is right adjoint to the endofunctor $-\times A$

$$
\begin{array}{lr}
\tau: \operatorname{Top}(S \times A, T) \rightarrow \operatorname{Top}\left(S, T^{A}\right) & \text { (the exponential law), } \\
\tau(f)=g, \quad f(x, a)=g(x)(a) & (x \in S, a \in A) .
\end{array}
$$


Inserting preorders, the preordered topological space $T^{A} \subset \operatorname{Top}(A, T)$ of orderpreserving maps gives a restriction of the previous bijection $\tau$

$$
\varphi: \operatorname{pTop}(S \times A, T) \rightarrow \operatorname{p} \operatorname{Top}\left(S, T^{A}\right) .
$$

Indeed, the map $f: S \times A \rightarrow T$ respects preorders if and only if it does so in each variable, separately; which means that every map $g(x)=f(x,-): A \rightarrow T$ belongs to $T^{A}$ and the mapping $g: X \rightarrow T^{A}$ respects preorders.

\section{Directed homotopy of inequilogical spaces}

This brief study is meant as a support for directed homology.

\subsection{Paths and symmetries}

A (directed) path in an inequilogical space $X$ is a map $a: \uparrow \mathbf{I} \rightarrow X$ defined on the standard ordered interval. The path $a$ has two endpoints in the underlying space $|X|$, or faces $\partial^{0}(a)=a(0), \partial^{1}(a)=a(1)$. Every point $x \in|X|$ has a degenerate path $0_{x}$, constant at $x$. Generally, paths are not reversible nor can be concatenated, as one can easily see in $\uparrow \mathbf{S}_{e}^{1}$.

Indeed, the reversion symmetry $\rho: \mathbf{I} \rightarrow \mathbf{I}(\rho(t)=1-t)$ used to reverse path and homotopies for topological and equilogical spaces disappears for the directed interval $\uparrow \mathbf{I}$, in p Top and $\mathbf{p E q} \mathbf{l}$; more precisely, it has a weak surrogate, the reflection $\rho: \uparrow \mathbf{I} \rightarrow \uparrow \mathbf{I}^{\mathrm{op}}$ which turns a path $a: \uparrow \mathbf{I} \rightarrow X$ into a path of the opposite structure, $a^{\mathrm{op}} \circ \rho: \uparrow \mathbf{I} \rightarrow X^{\mathrm{op}}$.

On the other hand, the interchange symmetry subsists

$$
s: \uparrow \mathbf{I}^{2} \rightarrow \uparrow \mathbf{I}^{2}, \quad s\left(t_{1}, t_{2}\right)=\left(t_{2}, t_{1}\right) .
$$

This behaviour, with respect to the 'Cartesian generators' of the symmetries of the $n$-dimensional cube, is similar to that of spaces with distinguished paths [13]. On the other hand, cubical sets are able to break all the intrinsic symmetries of topological spaces: given a cubical set $K$, an 'edge' in $K_{1}$ need not have any counterpart with reversed vertices, nor a 'square' in $K_{2}$ any counterpart with horizontal and vertical faces interchanged (as more completely discussed in [15], 1.1). While for inequilogical spaces (and spaces with distinguished paths), the choice of privileged directions is essentially determined at the 1-dimensional level, cubical sets also offer the possibility of higher dimensional choices.

\subsection{Directed homotopy}

The standard inequilogical interval $\uparrow \mathbf{I}$ also produces the (directed) cylinder functor and its right adjoint, the (directed) path functor, or cocylinder (by exponential, 1.8)

$$
\begin{array}{lc}
I: \mathrm{p} \mathbf{E q} \mathbf{l} \rightarrow \mathrm{p} \mathbf{E q} \mathbf{l}, & I(X)=X \times \uparrow \mathbf{I}, \\
P: \mathrm{pEq} \mathbf{\mathbf { I }} \rightarrow \mathrm{p} \mathbf{E q} \mathbf{l}, & P(Y)=Y^{\uparrow \mathbf{I}} .
\end{array}
$$

Identifying $X \times\{*\}=X$ and $Y^{\{*\}}=Y$, the faces of these functors are produced by the endpoints of the interval, $\partial^{\alpha}:\{*\} \rightarrow \uparrow \mathbf{I}(8)$

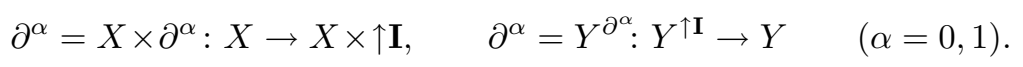


A (directed) homotopy $f: f_{0} \rightarrow f_{1}: X \rightarrow Y$ in pEql is defined as a map $f: X \times$ $\uparrow \mathbf{I} \rightarrow Y$ with faces $f \circ \partial^{\alpha}=f_{\alpha}$ (or, equivalently, $f: X \rightarrow Y^{\uparrow \mathbf{I}}$ with faces $\partial^{\alpha} \circ f=f_{\alpha}$ ). Paths correspond to the case $X=\{*\}$.

Again, these homotopies have no concatenation nor reversion. However, a homotopy in pEql produces a right homotopy in the category $\mathbf{C u b}$ of cubical sets (cf. $[\mathbf{1 5}], 1.6 .4)$

$$
\begin{gathered}
\square f: \square f_{0} \rightarrow_{R} \quad \square f_{1}: \square X \rightarrow \square Y, \\
\square_{n} f: \square_{n} X \rightarrow \square_{n+1} Y, \quad\left(\square_{n} f\right)(a)=f \circ(a \times \uparrow \mathbf{I}) .
\end{gathered}
$$

\subsection{Local maps and local homotopies}

In I.2.1 we introduced an extension of Eql, meant to simulate the local character of continuity; it produces a concatenation of the new paths (I.2) and the same homology (I.3.5).

Also here, it is interesting to extend $\mathrm{pEq}$ to the category $\mathrm{pEq} \mathbf{L}$ of inequilogical spaces and locally liftable mappings, or local maps. A local map $f: X \rightarrow Y$ (the arrow is marked with a dot) is a mapping $f:|X| \rightarrow|Y|$ between the underlying sets which admits an open saturated cover $\left(U_{i}\right)_{i \in I}$ of the space $X^{\sharp}$ (by open subsets, saturated for $\sim_{X}$ ), so that - for every index $i$ - the mapping $f$ has a partial (continuous, preorder-preserving) lifting $f_{i}: U_{i} \rightarrow Y^{\sharp}$

$$
f[x]=\left[f_{i}(x)\right], \quad \text { for } x \in U_{i} \text { and } i \in I .
$$

Equivalently, for every point $[x] \in|X|$, the mapping $f$ restricts to a map of inequilogical spaces on a suitable saturated neighbourhood $U$ of $x$ in $X^{\sharp}$.

Also here, all finite limits and arbitrary colimits of pEql still 'work' in the extension, which is thus cocomplete and finitely complete. A local isomorphism will be an isomorphism of $\mathbf{p E q L}$; a local (directed) path will be a local map $\uparrow \mathbf{I} \rightarrow X$; a local (directed) homotopy will be a local map $X \times \uparrow \mathbf{I} \rightarrow Y$, etc. Items of $\mathrm{pEq}$ will be called global (or also elementary, in the case of paths) when we want to distinguish them from the corresponding local ones.

Coming back to our models of the circle $(1.6,1.7)$, the canonical map $p: \uparrow \mathbf{S}_{e}^{1} \rightarrow$ $\uparrow \overline{\mathbf{S}}_{e}^{1}$ is not locally invertible: the topological inverse $\mathbf{R} / \mathbf{Z}=\mathbf{I} / \partial \mathbf{I}$ cannot be locally lifted at [0]; but, as in I.2.2, an inverse up to local homotopy exists.

By the local character of continuity in Top, the embedding Top $\subset$ pEqL is still full and reflective, with reflector (left adjoint) $|-|: \mathrm{pEqL} \rightarrow$ Top. Notice that the forgetful functor $|-|: \mathrm{pEq} \mathbf{\mathbf { l }} \rightarrow \mathrm{p}$ Top cannot be extended to local maps, since preserving preorder is not a local property, generally. Yet it becomes so when the domain $A$ of a map has a compact support $A^{\sharp}$; or, more generally, if in the preordered space $A^{\sharp}$ any two comparable points $x \prec_{A} y$ are contained in some compact subspace (as it happens in $\uparrow \mathbf{R}$ ). Therefore, as in I.2.7, a local path $a: \uparrow \mathbf{I} \rightarrow ; X$ is always a finite concatenation of elementary paths in $X$, up to local homotopy with fixed endpoints.

\subsection{The fundamental category}

Let $X$ be an inequilogical space, and $a, b: \uparrow \mathbf{I} \rightarrow X$ two consecutive local paths: $a(1)=x=b(0) \in|X|$. The concatenation $c=a * b: \uparrow \mathbf{I} \rightarrow X$ is defined in three steps 
(as in I.2.6, for equilogical spaces)

$$
c: \mathbf{I} \rightarrow|X|, \quad c(t)= \begin{cases}a(3 t), & \text { if } 0 \leqslant t \leqslant 1 / 3 \\ a(1)=b(0), & \text { if } 1 / 3 \leqslant t \leqslant 2 / 3 \\ b(3 t-2), & \text { if } 2 / 3 \leqslant t \leqslant 1,\end{cases}
$$

allowing for a stop at the concatenation point: this mapping is locally liftable (since, on the open subsets $[0,1 / 2[] 1 / 3,,2 / 3[] 1 / 2,1$,$] it essentially reduces to the given local$ directed paths or to a constant mapping, at the middle subset).

We have thus the fundamental category $\uparrow \Pi_{1}(X)$ of an inequilogical space: a vertex is a point $x \in|X|$ of the underlying set; an arrow $[a]: x \rightarrow y$ is an equivalence class of local paths from $x$ to $y$, up to local homotopy with fixed endpoints. Associativity is proved in the usual way (with slight adaptations due to the particular form of (22)); as well as the existence of identities (the classes of constant paths). Globally, we have a functor

$$
\uparrow \Pi_{1}: \mathrm{pEqL} \rightarrow \text { Cat. }
$$

The endomorphisms of $\uparrow \Pi_{1}(X)$ at a point $x_{0} \in|X|$ form the fundamental monoid $\uparrow \pi_{1}\left(X, x_{0}\right)$. Looking at the examples of 1.2 , it is evident that these monoids can contain far less information than the category $\uparrow \Pi_{1}(X)$, and also be trivial when the latter is not.

\subsection{Local homotopy invariance}

Local directed homotopies can be concatenated, but not reversed, generally. The directed homotopy type has to be defined taking this into account (as in [12] , 2.4, for spaces with distinguished paths).

For local maps $f, g: X \rightarrow Y$ in $\mathbf{p E q} \mathbf{L}$, the homotopy preorder $f \preceq g$ is defined by the existence of a local homotopy $f \rightarrow g$; it is consistent with composition $(f \preceq g$ and $f^{\prime} \preceq g^{\prime}$ imply $f^{\prime} f \preceq g^{\prime} g$ ) but not symmetric ( $f \preceq g$ is equivalent to $g^{\text {op }} \preceq f^{\text {op }}$ ). We shall write $f \simeq g$ the equivalence relation generated by $\preceq$ : there is a finite sequence $f \preceq f_{1} \succeq f_{2} \preceq f_{3} \ldots g$ (of local maps between the same objects); it is a congruence of categories. A local homotopy equivalence will be a local map $f: X \rightarrow Y$ having a homotopy inverse $g: Y \rightarrow X$, in the sense that $g f \simeq i d X, f g \simeq i d Y$. Then we write $X \simeq Y$, and say that they are locally homotopy equivalent, or have the same (directed) local homotopy type.

While the homotopy invariance of the fundamental groupoid of equilogical spaces (or of any undirected structure) works up to equivalence of groupoids, the homotopy invariance of the fundamental category is a more delicate question, as discussed in [12] for other directed structures. Without repeating the whole argument, let us note that a local homotopy $F: f \rightarrow g: X \rightarrow Y$ in $\mathbf{p E q L}$ produces a natural transformation $\uparrow \Pi_{1}(f) \rightarrow \uparrow \Pi_{1}(g)$ of the associated functors $\uparrow \Pi_{1}(X) \rightarrow \uparrow \Pi_{1}(Y)$ which need not be invertible; this is a (directed!) homotopy in Cat. Therefore, knowing that the inequilogical spaces $X, Y$ have the same directed homotopy type, only implies that the same is true of their fundamental categories, for a notion of directed homotopy equivalence in Cat, studied in [12], Section 4 (and defined as above for $\mathrm{p} \mathbf{E q} \mathbf{L}$ ); this relation is weaker than categorical equivalence but stronger 
than homotopy equivalence of the classifying spaces, which is not a directed notion.

\section{Directed homology of inequilogical spaces}

In I.3 we have studied the extension of singular homology to equilogical spaces. We show now that inequilogical spaces have a directed homology, formed of preordered abelian groups.

\subsection{Directed homology of cubical sets}

We have already recalled how cubical sets break both the reversion and interchange symmetry (2.1). Their directed homology, introduced and studied in [15], is obtained by enriching their ordinary homology groups with a natural preorder, generated by taking the given cubes as positive.

More precisely, given a cubical set $K$, take the $n$-th component of its (normalised) chain complex, i.e. the free abelian group generated by the non degenerate $n$-cubes of $K$

$$
C_{n}(K)=\mathbf{Z} \bar{K}_{n} \quad\left(\bar{K}_{n}=K_{n} \backslash \operatorname{Deg}_{n} K\right),
$$

and write it as $\uparrow C_{n}(K)$ when ordered by the positive cone of positive chains $\mathbf{N} \bar{K}_{n}$. (Note that the differential $\partial_{n}: C_{n}(K) \rightarrow C_{n-1}(K)$ does not preserve this order, generally.)

The directed homology of a cubical set is its ordinary homology, equipped with the preorder induced by the order of $\uparrow C_{n}(K)$ on its homology subquotient, $\operatorname{Ker} \partial_{n} / \operatorname{Im} \partial_{n+1}$; we have functors

$$
\uparrow H_{n}: \mathbf{C u b} \rightarrow \mathrm{d} \mathbf{A b}, \quad \uparrow H_{n}(K)=\uparrow H_{n}\left(\uparrow C_{*}(K)\right),
$$

with values in the category $\mathrm{d} \mathbf{A} \mathbf{b}$ of preordered abelian groups and preorder-preserving homomorphisms. In particular, the free abelian group $\uparrow H_{0}(K)$ is ordered, with positive cone generated by the homology classes of the vertices of $K$.

Forgetting preorders, one gets the usual chain and homology functors, $C_{*}(K)$ and $H_{*}(K)$.

Notice that, when $K=\square X$ is the singular cubical set of a topological space, forgetting preorders does not likely destroy any essential information. First, $\uparrow H_{0}(\square X)$ has the obvious order described above; then, the preorder of $\uparrow H_{1}(\square X)$ is necessarily chaotic: every homology class belongs to the positive cone. (Indeed, for every 1-cube $a: \mathbf{I} \rightarrow X$, the reversed path $a \rho$ is equivalent to the chain $-a$, modulo boundaries). It would be interesting to prove a similar result in higher dimension.

\subsection{Directed homology of inequilogical spaces}

Now, an inequilogical space $X$ (on a preordered space $X^{\sharp}=(T, \prec)$ ) has a cubical set of singular cubes (produced by the cocubical set of standard ordered cubes $\uparrow \mathbf{I}^{n}$, their faces and degeneracies)

$$
\square: \mathrm{pEql} \rightarrow \mathbf{C u b}, \quad \square_{n} X=\operatorname{pEql}\left(\uparrow \mathbf{I}^{n}, X\right)=\left(\square_{n} X^{\sharp}\right) / \sim_{n},
$$

whose $n$-component 'is' the quotient of $\square_{n} X^{\sharp}=\operatorname{p} \mathbf{T o p}\left(\uparrow \mathbf{I}^{n}, X^{\sharp}\right)$ modulo the equivalence relation $\sim_{n}$ obtained by projecting cubes along the canonical projection 
$X^{\sharp} \rightarrow|X|=X^{\sharp} / \sim$. Notice that $\square X$ is a subobject of the cubical set of the underlying equilogical space $(T, \sim)$

$$
\square_{n} X \subset \square_{n}(T, \sim)=\operatorname{Eql}\left(\mathbf{I}^{n},(T, \sim)\right) .
$$

This canonical embedding of pEql in Cub defines the singular homology of inequilogical spaces, again as a sequence of preordered abelian groups:

$$
\uparrow H_{n}: \mathrm{pEq} \mathbf{l} \rightarrow \mathrm{d} \mathbf{A b}, \quad \uparrow H_{n}(X)=\uparrow H_{n}(\square X),
$$

and a map of inequilogical spaces induces preorder-preserving homomorphisms. This functor is homotopy invariant: given a homotopy $f: f_{0} \rightarrow f_{1}$, we have $\uparrow H_{n}\left(f_{0}\right)=$ $\uparrow H_{n}\left(f_{1}\right)$, as it follows immediately from the homotopy between the corresponding morphisms of cubical sets (cf. (20)).

If $X$ is an equilogical space (with the coarse preorder), the cubical set $\square X$ is precisely the one already considered in Part I, and the singular homology groups are - algebraically - the same, while their preorder is likely of no interest.

But notice that, in the general case, the groups $\uparrow H_{n}(X)$ can differ - even algebraically - from the groups $H_{n}(T, \sim)$ of the underlying equilogical space; as a trivial example, if the preorder $\prec_{X}$ is discrete (the equality), all directed cubes $\uparrow \mathbf{I}^{n} \rightarrow X$ are constant and $\uparrow H_{n}(X)=0$ for $n>0$. In Section 6 we will see various inequilogical tori, with the classical homology groups and different preorders.

\subsection{Local directed homology}

Extending the results of I.3, the wider category $\mathrm{pEqL}$ of local maps (2.3) gives the local (directed) cubes $a: \uparrow \mathbf{I}^{n} \rightarrow X$, the directed complex of local chains $\uparrow C L_{*}(X)$ and the preordered groups $\uparrow H L_{n}(X)$ of local directed homology

$$
\begin{array}{cc}
\uparrow \square L_{n} X=\mathrm{pEq} \mathbf{L}\left(\mathbf{I}^{n}, X\right), & \uparrow C L_{*}(X)=\uparrow C_{*}(\uparrow \square L X), \\
\uparrow H L_{n}: \mathrm{p} \mathbf{E q} \mathbf{L} \rightarrow \mathrm{d} \mathbf{A b}, & \uparrow H L_{n}(X)=\uparrow H_{n}\left(\uparrow C L_{*}(X)\right) .
\end{array}
$$

The functors $\uparrow H L_{n}$ are invariant by local directed homotopy: as in I.3.3, a local directed homotopy $f \prec g$ gives $\uparrow H L_{n}(f)=\uparrow H L_{n}(g)$.

Now, as in I.3.5, the local homology $\uparrow H L_{n}(X)$ always coincides with the global homology $\uparrow H_{n}(X)$; more precisely, the embedding $\uparrow C_{*}(X) \subset \uparrow C L_{*}(X)$ induces an isomorphism $\uparrow H_{n}(X) \cong \uparrow H L_{n}(X)$, natural for global maps. Thus, global homology is also invariant for local homotopy, and locally homotopy equivalent objects have the same directed homology, up to isomorphism of preordered abelian groups.

\subsection{Properties of directed homology}

The algebraic properties work as in the non-directed case (I.3); but one should take care of the fact that preorder is not respected by the differential of our directed chain complexes (3.1), which produces other anomalies (as in the directed homology of cubical sets $[\mathbf{1 5}])$.

We have already seen the homotopy invariance of global and local directed homology, as well as their coincidence. The Mayer-Vietoris sequence works as in I.3.8, taking into account that its differential does not preserve preorders (as for cubical sets [15]); on the other hand, excision works well (as in I.3.8) and gives an isomorphism of preordered abelian groups. 
Exceptionally, suspension works worse than for cubical sets (cf. 3.5).

\subsection{Computations}

The previous results allow one to compute easily the algebraic part of directed homology; then, its preorder has often to be computed by a concrete inspection of the directed cubes of a given inequilogical space.

Thus, it is easy to prove, using the Mayer-Vietoris sequence, that the directed homology of the inequilogical spheres $\uparrow \mathbf{S}_{e}^{n}$ or $\uparrow \overline{\mathbf{S}}_{e}^{n}$ yields the usual algebraic groups. And we already know that their ordered group $\uparrow H_{0}$ is always $\uparrow \mathbf{Z}$, for $n>0$ (3.1).

Now, for $n=1$, all the directed paths $a: \uparrow \mathbf{I} \rightarrow \uparrow \overline{\mathbf{S}}_{e}^{1}$ move 'counterclockwise' around the circle, and every positive cycle is homologous to turning around 'counterclockwise' $k$ times, for some $k \in \mathbf{N}$. In other words (recalling that $\uparrow \mathbf{S}_{e}^{1}$ and $\uparrow \overline{\mathbf{S}}_{e}^{1}$ are locally homotopy equivalent, 2.3 )

$$
\uparrow H_{1}\left(\uparrow \mathbf{S}_{e}^{1}\right)=\uparrow H_{1}\left(\uparrow \overline{\mathbf{S}}_{e}^{1}\right)=\uparrow \mathbf{Z} .
$$

The results on the higher spheres are less interesting: for all $n \geqslant 2, \uparrow H_{n}\left(\uparrow \mathbf{S}_{e}^{n}\right)$ is the group of integers with the chaotic preorder. In fact, a positive generator of $\uparrow H^{2}\left(\uparrow \mathbf{S}_{e}^{2}\right)$ is the 2-cube $a: \uparrow \mathbf{I}^{2} \rightarrow\left(\uparrow \mathbf{I}^{2}, \partial \mathbf{I}^{2}\right)$ induced by the identity of the ordered square. But, using the interchange of coordinates $\sigma: \uparrow \mathbf{I}^{2} \rightarrow \uparrow \mathbf{I}^{2}$ (17), we get another positive cycle $a \sigma$, showing that the opposite homology class $[a \sigma]=-[a]$ is (weakly) positive as well. In higher dimension, use $\sigma \times \uparrow \mathbf{I}^{n-2}$.

This also shows that, in contrast with cubical sets, directed homology of inequilogical spaces does not agree with suspension (cf. [15], Section 5). As we have seen, these drawbacks are directly linked with the fact that the interchange symmetry $\sigma$ subsists in pEql: the directed structure of inequilogical spaces distinguishes directed paths in an effective way, but can only distinguish higher cubes through directed paths; this is not sufficient to get good results for $\uparrow H_{k}$, with $k>1$.

\subsection{Inequilogical realisation}

We have seen in I.5.6 that a cubical set has an equilogical realisation, yielding the left adjoint $\mathcal{E}: \mathbf{C u b} \rightarrow \mathbf{E q} \mathbf{l}$ to the functor $\square: \mathbf{E q l} \rightarrow \mathbf{C u b}$. Enriching its support with the standard order, we obtain the inequilogical realisation functor

$$
\uparrow \mathcal{E}: \mathbf{C u b} \rightarrow \mathrm{pEq}, \quad \uparrow \mathcal{E}(K)=\left(\sum_{a} \uparrow \mathbf{I}^{n(a)}, \sim\right),
$$

left adjoint to $\square: \mathrm{pEq} \mathbf{\mathbf { l }} \rightarrow \mathbf{C u b}$ (cf. (26)).

As in the non-directed case, the sum is indexed on all cubes $a$ of $K$, of which $n(a)$ is the dimension; the equivalence relation $\sim$ (analytically described in I.5.6.2) is generated by identifying points along faces and degeneracies. Thus, the usual topological realisation ('geometric realisation') $\mathcal{R}(K)$ is precisely the space underlying the equilogical (and inequilogical) realisation

$$
\mathcal{R}(K)=\left(\sum_{a} \mathbf{I}^{n(a)}\right) / \sim=|\mathcal{E}(K)| .
$$

(We have also proved, in I.5.7, that these objects - $\mathcal{R}(K)$ and $\mathcal{E}(K)$ - are locally homotopically equivalent.) As in I.5.9, the realisation (31) can be simplified, up to 
isomorphism, omitting all cubes $a$ which are degenerate; moreover, for a finitely generated cubical set $K$, one can also omit those cubes which are faces of a nondegenerate cube.

Taking this reduction into account, one easily sees that the standard inequilogical circle $\uparrow \mathbf{S}_{e}^{1}=\left(\uparrow \mathbf{I}, R_{\partial \mathbf{I}}\right)$ is (isomorphic to) the inequilogical realisation of the directed cubical circle $\uparrow \mathbf{s}^{1}=\langle * \rightarrow *\rangle$, generated by one vertex and one edge. More generally, the $k$-gonal inequilogical circle $\uparrow C_{k}=\left(k \uparrow \mathbf{I}, R_{k}\right)$ resulting from the sum $\uparrow \mathbf{I}+\ldots+\uparrow \mathbf{I}$ of $k$ copies of the directed interval (in pTop), together with the equivalence relation $R_{k}$ identifying the terminal point of any addendum with the initial point of the following one, circularly (cf. I.1.4.4) is the inequilogical realisation of the directed $k$-gonal cubical circle $\uparrow c_{k}$ (generated by $k$ vertices and $k$ edges, with obvious faces).

\section{Formal quotients as cubical sets or equilogical spaces}

Equilogical and inequilogical spaces can express 'formal quotients' of spaces, of interest in noncommutative geometry; but the second structure can reach finer results.

\subsection{Actions on preordered spaces}

Let $(X, \prec)$ be a preordered space on which the group $G$ acts (all its operators $X \rightarrow X$ preserve the preorder), so that $G$ also acts on the cubical subset $\square(X, \prec) \subset$ $\square X$ of preorder-preserving cubes $\uparrow \mathbf{I}^{n} \rightarrow(X, \prec)$.

We have already seen in [G4] that the directed orbit cubical set $\square(X, \prec) / G$ can be much more relevant than the ordinary orbit space $X / G$ or the undirected orbit cubical set $(\square X) / G$ (examples are recalled below). We show now that the orbit inequilogical space $\left(X, \prec, \equiv_{G}\right)$ can often give the same results as the directed cubical structure, $\square(X, \prec) / G$.

We say that the action of the group $G$ on the space $X$ is pathwise free (I.4.1) if, whenever two paths $a, b: \mathbf{I} \rightarrow X$ have the same projection to the orbit space $X / G$, there is precisely one $g \in G$ such that $a=b+g$; then, of course, the same works for all pairs of $n$-cubes $a, b: \mathbf{I}^{n} \rightarrow X$, so that the canonical surjection

$$
(\square X) / G \rightarrow \square\left(X, \equiv_{G}\right),
$$

is an isomorphism of cubical sets. We have seen that a proper action is always pathwise free (I.4.2a), while (obviously) a pathwise free one is necessarily free.

Now, for a pathwise free action of the group $G$ on the preordered space $(X, \prec)$, the isomorphism (33) restricts to an isomorphism of cubical sets - whence of their directed homology groups

$$
\square\left(X, \prec, \equiv_{G}\right)=\square(X, \prec) / G, \quad \uparrow H_{n}\left(X, \prec, \equiv_{G}\right)=\uparrow H_{n}(\square(X, \prec) / G)
$$

\subsection{Inequilogical spaces and irrational rotations}

In particular, we can apply this to a well-known situation, related to the irrational rotation $\mathrm{C}^{*}$-algebras (as recalled in I.4): the action of the group $G_{\vartheta}=\mathbf{Z}+\vartheta \mathbf{Z}(\vartheta$ irrational) on the real line, by translations. 
Take the cubical set $\square \uparrow \mathbf{R}$ of all order-preserving maps $\mathbf{I}^{n} \rightarrow \mathbf{R}$, and consider the irrational rotation cubical sets $C_{\vartheta}=(\square \uparrow \mathbf{R}) / G_{\vartheta}$. Algebraically, the homology groups are independent of $\vartheta$, but directed homology gives a finer information

$$
\uparrow H_{1}\left(C_{\vartheta}\right) \cong \uparrow G_{\vartheta},
$$

as a (totally) ordered subgroup of $\mathbf{R}$ ([15], Thm. 4.8), which gives a strong information on $\vartheta$. It follows that the cubical sets $C_{\vartheta}$ have the same classification up to isomorphism [G4, Thm. 4.9] as the $\mathrm{C}^{*}$-algebras $A_{\vartheta}$ up to strong Morita equivalence: $\vartheta$ is determined up to the action of the linear group $\mathrm{GL}(2, \mathbf{Z})$ (I.4.4.1).

This example shows that the ordering of directed homology can carry a relevant information. Further, comparison with the stricter classification of the algebras $A_{\vartheta}$ up to isomorphism $([\mathbf{1 5}], 4.1)$ shows that cubical sets provide a sort of 'noncommutative topology', without the metric character of noncommutative geometry. (Normed cubical sets, studied in [16], have such a character.)

We show now that the same holds for the irrational rotation inequilogical space

$$
C_{\vartheta}^{\prime}=\left(\uparrow \mathbf{R}, \equiv_{G_{\vartheta}}\right)=\left(\mathbf{R}, \leqslant, \equiv_{G_{\vartheta}}\right) .
$$

4.3 Proposition. We have

$$
\uparrow H_{1}\left(C_{\vartheta}^{\prime}\right)=\uparrow H_{1}\left((\square \uparrow \mathbf{R}) / G_{\vartheta}\right) \cong \uparrow G_{\vartheta} .
$$

Proof. The action of $G_{\vartheta}$ on the (ordered) line is pathwise free, as it follows immediately from the fact that $G_{\vartheta}$ is a totally disconnected subgroup of $\mathbf{R}$ (if the paths $a, b: \mathbf{I} \rightarrow X$ have the same projection to $X / G_{\vartheta}$, their difference $a-b: \mathbf{I} \rightarrow G_{\vartheta}$ must be constant). Therefore, by (34), the result on the directed homology of the cubical set $C_{\vartheta}=(\square \uparrow \mathbf{R}) / G_{\vartheta}$ can also be stated in terms of the orbit inequilogical space $C_{\vartheta}^{\prime}$.

4.4 Theorem (Classification Theorem, I). The following conditions on the irrational numbers $\vartheta, \zeta$ are equivalent:

(a) the inequilogical spaces $C_{\vartheta}^{\prime}=\left(\uparrow \mathbf{R}, \equiv_{G_{\vartheta}}\right)$ and $C_{\zeta}^{\prime}$ are isomorphic;

(b) the $C^{*}$-algebras $A_{\vartheta}$ and $A_{\zeta}$ are strongly Morita equivalent;

(c) the cubical sets $C_{\vartheta}=(\square \uparrow \mathbf{R}) / G_{\vartheta}$ and $C_{\zeta}$ are isomorphic;

(d) the ordered groups $\uparrow G_{\vartheta}$ and $\uparrow G_{\zeta}$ are isomorphic;

(e) $\vartheta$ and $\zeta$ are conjugate under the action of $\mathrm{GL}(2, \mathbf{Z})($ I.4.4.1);

(f) $\zeta$ belongs to the closure of $\vartheta$ under the transformations $R(t)=t^{-1}$ and $T^{ \pm 1}(t)=$ $t \pm 1$, on $\mathbf{R} \backslash \mathbf{Q}$.

Proof. The equivalence of properties (b) and (e) is a combined result of Pimsner - Voiculescu [23] and Rieffel [25]; that of (c) - (f) has been proved in [15], Thm. 4.9. Moreover, (a) implies (d) by Proposition 4.3, applying the directed homology group $\uparrow H_{1}$. Finally, to deduce (a) from (f), it suffices to consider the cases $\zeta=\vartheta+k$ $(k \in \mathbf{Z})$ and $\zeta=\vartheta^{-1}$. In the first case, the ordered groups $\uparrow G_{\vartheta}$ and $\uparrow G_{\zeta}$ coincide (as well as their action on $\uparrow \mathbf{R})$; in the second $\left(\zeta=\vartheta^{-1}\right)$, the isomorphism of preordered topological spaces

$$
f: \uparrow \mathbf{R} \rightarrow \uparrow \mathbf{R}, \quad f(t)=|\vartheta| . t,
$$


restricts to an isomorphism $f^{\prime}: \uparrow G_{\vartheta} \rightarrow \uparrow G_{\zeta}$, obviously consistent with the actions $\left(f(t+g)=f(t)+f^{\prime}(g)\right)$, and induces an isomorphism of inequilogical spaces $\left(\uparrow \mathbf{R}, \equiv_{G_{\vartheta}}\right) \rightarrow\left(\uparrow \mathbf{R}, \equiv_{G_{\zeta}}\right)$.

\section{Higher dimensional noncommutative tori}

The classification theorem 4.4 is extended to the inequilogical spaces $C_{\vartheta}^{\prime}=$ $\left(\uparrow \mathbf{R}, \equiv_{G_{\vartheta}}\right)$, where $\vartheta$ is an $n$-tuple of real numbers linearly independent on $\mathbf{Q}$.

\subsection{The extension}

Take now an n-tuple of real numbers $\vartheta=\left(\vartheta_{1}, \ldots, \vartheta_{n}\right)$, linearly independent on the rationals, and consider the additive subgroup $G_{\vartheta}=\sum_{i} \vartheta_{i} \mathbf{Z} \cong \mathbf{Z}^{n}$ of the real line. (The previous case corresponds to the pair $(1, \vartheta)$.)

Again, the (totally disconnected) group $G_{\vartheta}$ acts pathwise freely on the directed line and on the cubical set $\square \uparrow \mathbf{R}$. It was proved in [15], 4.4, that the directed 1homology of the cubical set $(\square \uparrow \mathbf{R}) / G_{\vartheta}$ gives back the total order of $\uparrow G_{\vartheta}$ (as a subgroup of the ordered real line). As a consequence (by (36)), the same holds for the orbit inequilogical space $\left(\uparrow \mathbf{R}, \equiv_{G_{\vartheta}}\right)$

$$
\uparrow H_{1}\left(\uparrow \mathbf{R}, \equiv_{G_{\vartheta}}\right)=\uparrow H_{1}\left((\square \uparrow \mathbf{R}) / G_{\vartheta}\right)=\uparrow G_{\vartheta}=\uparrow\left(\sum_{i} \vartheta_{i} \mathbf{Z}\right) .
$$

\subsection{Integral matrices}

We shall use the group $\mathrm{GL}(n, \mathbf{Z})$ of matrices with integral entries and determinant \pm 1 , with its natural action (on the right) on $\mathbf{R}^{n}$ (and $\mathbf{Z}^{n}$ ).

Let us recall that $\mathrm{GL}(n, \mathbf{Z})$ admits the following finite system of generators ([30], p. 145):

(a) diagonal matrices with entries \pm 1 ;

(b) permutation matrices (all entries are 0 except precisely one in each row and one in each column, which is equal to 1 );

(c) upper triangular matrices with 1 on the diagonal and all the elements above equal to 0 , except one of them which is equal to 1 .

Therefore, the action of $\mathrm{GL}(n, \mathbf{Z})$ on $\mathbf{R}^{n}$ is generated by the following transformations:

(i) change of sign of one coordinate (an action of the group $(\mathbf{Z} / 2)^{n}$ ),

(ii) permutation of coordinates (an action of the symmetric group $S_{n}$ ),

(iii) $T_{i j}\left(t_{1}, \ldots, t_{n}\right)=\left(t_{1}, \ldots, t_{i}+t_{j}, \ldots, t_{j}, \ldots, t_{n}\right) \quad($ for $1 \leqslant i<j \leqslant n)$.

It is sufficient to consider finite composites of these transformations, since also the inverse of $T_{i j}$ can be expressed as such a composite: $T_{i j}^{-1}\left(t_{1}, \ldots, t_{n}\right)=\left(t_{1}, \ldots, t_{i}-\right.$ $\left.t_{j}, \ldots, t_{j}, \ldots, t_{n}\right)$.

This action is extended to the group $\operatorname{GL}(n, \mathbf{Z}) \times \mathbf{R}_{*}^{+}$, where a real number $\lambda>0$ acts on $\mathbf{R}^{n}$ by multiplication

(iv) $\lambda .\left(t_{1}, \ldots, t_{n}\right)=\left(\lambda t_{1}, \ldots, \lambda t_{n}\right)$. 
Given an $n$-tuple $t \in \mathbf{R}^{n}$, we shall denote by $t^{\wedge}$ its closure under the action of the group $\mathrm{GL}(n, \mathbf{Z})$, or equivalently under the transformations of type (i)-(iii); by $t^{\wedge}$ its closure under the action of $\mathrm{GL}(n, \mathbf{Z}) \times \mathbf{R}_{*}^{+}$, or equivalently under the transformations of type (i)-(iv).

5.3 Lemma. Let $\vartheta, \zeta$ be $n$-tuples of real numbers, linearly independent on $\mathbf{Q}$. Then the following conditions are equivalent:

(a) the groups $G_{\vartheta}=\sum_{i} \vartheta_{i} \mathbf{Z}$ and $G_{\zeta}$ coincide, as subsets of the line,

(b) $\vartheta$ and $\zeta$ are conjugate under the action of $\mathrm{GL}(n, \mathbf{Z})$,

(c) $\zeta$ belongs to the closure $\vartheta^{\wedge}$ of $\vartheta$ under the transformations (i)-(iii) of 5.2.

Proof. The last two conditions are equivalent, by 5.2. Assuming that $G_{\vartheta}=G_{\zeta}$, we can write $\zeta=\vartheta A$ and $\vartheta=\zeta B$, with matrices $A, B \in M_{n}(\mathbf{Z})$. Therefore $\vartheta\left(A B-I_{n}\right)=$ 0 , which (by our hypotheses on $\vartheta$ ) implies $A B=I_{n}$; similarly for $B A$, and (b) holds. Finally, to prove that (c) implies (a), it suffices to consider that, whenever $\zeta$ is obtained from $\vartheta$ by one of the transformations (i)-(iii) of $5.2, \uparrow G_{\vartheta}$ and $\uparrow G_{\zeta}$ coincide.

5.4 Theorem (Classification Theorem, II). Let $\vartheta, \zeta$ be $n$-tuples of real numbers, linearly independent on $\mathbf{Q}$. The following conditions are equivalent:

(a) the inequilogical spaces $C_{\vartheta}^{\prime}=\left(\uparrow \mathbf{R}, \equiv_{G_{\vartheta}}\right)$ and $C_{\zeta}^{\prime}$ are isomorphic;

(b) the cubical sets $C_{\vartheta}=(\square \uparrow \mathbf{R}) / G_{\vartheta}$ and $C_{\zeta}$ are isomorphic;

(c) the ordered groups $\uparrow G_{\vartheta}$ and $\uparrow G_{\zeta}$ are isomorphic;

(d) $\vartheta$ and $\zeta$ are conjugate under the action of $\operatorname{GL}(n, \mathbf{Z}) \times \mathbf{R}_{*}^{+}$(5.2),

(e) $\zeta$ belongs to the closure $\vartheta^{n}$ of $\vartheta$ under the transformations (i)-(iv) of 5.2.

Proof. The conditions (d), (e) are equivalent, by 5.2. Moreover, (a) trivially implies (b), which implies (c), since we already know that the ordered homology group $\uparrow H_{1}\left(\square \uparrow \mathbf{R} / G_{\vartheta}\right)$ is isomorphic to $\uparrow G_{\vartheta}$ (cf. (39)). To prove that (e) implies (a), it suffices to consider four cases, where $\zeta$ is obtained from $\vartheta$ by one of the transformations (i)-(iv) of 5.2. In the first three cases, $\uparrow G_{\vartheta}$ and $\uparrow G_{\zeta}$ coincide (as well as their action on $\uparrow \mathbf{R}$ ), whence $C_{\vartheta}^{\prime}=C_{\zeta}^{\prime}$. In the fourth, $\zeta=\lambda \vartheta$ (with $\lambda>0$ ) and the isomorphism of ordered topological spaces

$$
f: \uparrow \mathbf{R} \rightarrow \uparrow \mathbf{R}, \quad f\left(t_{1}, \ldots, t_{n}\right)=\left(\lambda t_{1}, \ldots, \lambda t_{n}\right),
$$

restricts to an isomorphism of ordered groups $f^{\prime}: \uparrow G_{\vartheta} \rightarrow \uparrow G_{\zeta}$, obviously consistent with the actions $\left(f(a+g)=f(a)+f^{\prime}(g)\right)$. Finally, it induces an isomorphism of inequilogical spaces $C_{\vartheta}^{\prime}=C_{\zeta}^{\prime}$.

We are left with proving that (c) implies (e). Let us take two sequences $\vartheta, \zeta$ such that $\uparrow G_{\vartheta} \cong \uparrow G_{\zeta}$ and prove that $z \in \vartheta^{\varkappa}$. Operating with transformations of type 5.2(i) (changing the sign of one component), we can assume that all the components of $\vartheta$ and $\zeta$ are positive.

Let us begin noting that the sequence $\vartheta$ (linearly independent on the rationals) defines an algebraic isomorphism $\mathbf{Z}^{n} \cong G_{\vartheta}$, which becomes an order isomorphism for the ordered group $\uparrow_{\vartheta} \mathbf{Z}^{n}$

$$
\begin{aligned}
\uparrow_{\vartheta} \mathbf{Z}^{n} \rightarrow & \uparrow G_{\vartheta}, \quad\left(a_{1}, \ldots, a_{n}\right) \mapsto(a \mid \vartheta)=\sum_{i} a_{i} \vartheta_{i}, \\
& \left(a_{1}, \ldots, a_{n}\right)>\vartheta \\
0 & \Leftrightarrow(a \mid \vartheta)>0 .
\end{aligned}
$$


It will be useful to note that this order determines the (positive) sequence $\vartheta$ up to a multiplicative scalar $\lambda=\vartheta_{n}>0$, by the following upper bounds in $\mathbf{R}$ (for $i<n)$

$$
\vartheta_{i} / \vartheta_{n}=\sup \left\{-a_{n} / a_{i} \mid\left(0, \ldots, a_{i}, 0, \ldots, a_{n}\right)>{ }_{\vartheta} 0, a_{i}>0\right\} .
$$

Now, our isomorphism $\uparrow G_{\vartheta} \cong \uparrow G_{\zeta}$ produces an isomorphism $f: \uparrow_{\vartheta} \mathbf{Z}^{n} \rightarrow \uparrow_{\zeta} \mathbf{Z}^{n}$. The underlying algebraic isomorphism $f: \mathbf{Z}^{n} \rightarrow \mathbf{Z}^{n}$ can be factored as $f=f_{m} \ldots f_{1}$, with factors as below (cf. 5.2)

$$
\begin{array}{cr}
\rho_{i}(a)=\left(a_{1}, \ldots,-a_{i}, \ldots, a_{n}\right) & (i=1, \ldots, n), \\
\sigma(a)=\left(a_{\tau 1}, \ldots, a_{\tau n}\right) & \left(\sigma \in S_{n}, \tau=\sigma^{-1}\right), \\
\tau_{i j}(a)=\left(a_{1}, \ldots, a_{i}+a_{j}, \ldots, a_{n}\right) & (1 \leqslant i<j \leqslant n) .
\end{array}
$$

Moreover $\rho_{i}$ (resp. $\sigma, \tau_{i j}$ ) is an order isomorphism $\uparrow_{\vartheta} \mathbf{Z}^{n} \rightarrow \uparrow_{\omega} \mathbf{Z}^{n}$, for a suitable $\omega \in \vartheta^{\wedge}$

$$
\begin{array}{rrr}
a>_{\vartheta} 0 \Leftrightarrow\left(\rho_{i}(a) \mid \rho_{i}(\vartheta)\right)>0 \Leftrightarrow \rho_{i}(a)>_{\omega} 0 & \left(\omega=\rho_{i}(\vartheta)\right), \\
a>_{\vartheta} 0 \Leftrightarrow(\sigma(a) \mid \sigma(\vartheta))>0 \Leftrightarrow \sigma(a)>_{\omega} 0 & (\omega=\sigma(\vartheta)), \\
a>_{\vartheta} 0 \Leftrightarrow \tau_{i j}(a)>_{\omega} 0 & \left(\omega=\left(\vartheta_{1}, \ldots, \vartheta_{i}, \ldots, \vartheta_{j}-\vartheta_{i}, \ldots, \vartheta_{n}\right)\right) .
\end{array}
$$

Thus, the isomorphism $f: \uparrow_{\vartheta} \mathbf{Z}^{n} \rightarrow \uparrow_{\zeta} \mathbf{Z}^{n}$ is also an iso $\uparrow_{\vartheta} \mathbf{Z}^{n} \rightarrow \uparrow_{\omega} \mathbf{Z}^{n}$ for a suitable $\omega \in \vartheta^{\wedge}$, and $\uparrow_{\zeta} \mathbf{Z}^{n}=\uparrow_{\omega} \mathbf{Z}^{n}$. By (42), $\zeta=\lambda \omega$ for some positive $\lambda$, and the thesis holds.

\section{Linear orders and inequilogical tori}

Each linear preorder on the vector space $\mathbf{R}^{n}$ produces a directed structure on the equilogical torus $\left(\mathbf{R}^{n}, \equiv_{\mathbf{Z}^{n}}\right)$, which can be analysed with directed homology.

\subsection{Linear preorders}

We shall use the following model of equilogical torus

$$
\overline{\mathbf{T}}_{e}^{n}=\left(\mathbf{R}^{n}, \equiv_{\mathbf{Z}^{n}}\right)=\overline{\mathbf{S}}_{e}^{1} \times \ldots \times \overline{\mathbf{S}}_{e}^{1},
$$

which is (by I.2.3) locally isomorphic to the topological space $\mathbf{T}^{n}=\mathbf{R}^{n} / \mathbf{Z}^{n}=$ $\mathbf{S}^{1} \times \ldots \times \mathbf{S}^{1}$. Enriching the support $\mathbf{R}^{n}$ with a preorder $\leqslant_{\Gamma}$, we get a family of inequilogical spaces

$$
\uparrow_{\Gamma} \mathbf{T}^{n}=\left(\mathbf{R}^{n}, \leqslant_{\Gamma}, \mathbf{Z}^{n}\right)
$$

which can be investigated with directed homology, and often classified.

All the preorders $\leqslant_{\Gamma}$ we will consider on $\mathbf{R}^{n}$ respect its linear structure and are - as a consequence - determined by a positive cone $\Gamma$ (closed under sum and multiplication by real scalars $l \geqslant 0$, hence convex). It will be important to assume that $\Gamma$ has internal points, as in all the planar examples below 

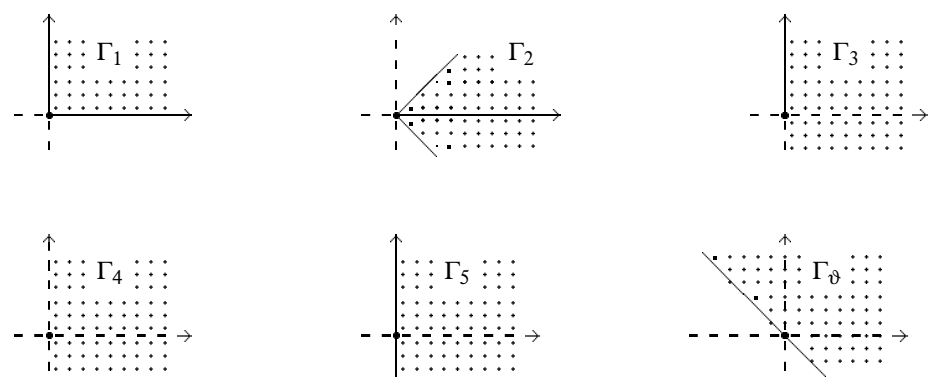

$$
\begin{array}{lll}
\Gamma_{1}=\left\{(x, y) \in \mathbf{R}^{2} \mid x \geqslant 0, y \geqslant 0\right\}, & & \Gamma_{2}=\{(x, y)|x \geqslant| y \mid\}, \\
\Gamma_{3}=\{(x, y) \mid x>0 ; y>0 \text { or } x=0 \leqslant y\}, & & \\
\Gamma_{4}=\{(x, y) \mid x>0 \text { or } x=0=y\}, & & \Gamma_{5}=\{(x, y) \mid x \geqslant 0\}, \\
\Gamma_{\vartheta}=\{(x, y) \mid x+\vartheta y \geqslant 0\} & & (\vartheta \text { irrational }) .
\end{array}
$$

The first three examples are 'vector lattices', also called Riesz spaces $[\mathbf{3}, \mathbf{2 0}])$ : in the first case we have the product order and in the third the lexicographic one (a total order). The fourth example is ordered but not a lattice; the last two are just preordered.

6.2 Theorem. Assuming that $\Gamma$ (a positive cone of the vector space $\mathbf{R}^{n}$ ) has internal points, the algebraic homology groups of the inequilogical torus $\uparrow_{\Gamma} \mathbf{T}^{n}=\left(\mathbf{R}^{n}, \leqslant_{\Gamma}\right.$ ,$\left.\equiv \mathbf{Z}^{n}\right)$ are the usual ones, and the inclusion

$$
\square\left(\uparrow_{\Gamma} \mathbf{T}^{n}\right)=\square\left(\mathbf{R}^{n}, \leqslant_{\Gamma}\right) / \mathbf{Z}^{n} \subset \square\left(\overline{\mathbf{T}}_{e}^{n}\right)=\left(\square \mathbf{R}^{n}\right) / \mathbf{Z}^{n},
$$

induces isomorphism in homology

$$
H_{k}\left(\uparrow_{\Gamma} \mathbf{T}^{n}\right) \cong H_{k}\left(\overline{\mathbf{T}}_{e}^{n}\right) \cong \mathbf{Z}^{\left(\begin{array}{l}
n \\
k
\end{array}\right)} \quad(0 \leqslant k \leqslant n) .
$$

Moreover

$$
\uparrow H_{1}\left(\uparrow_{\Gamma} \mathbf{T}^{n}\right) \cong\left(\mathbf{Z}^{n}, \leqslant_{\Gamma}\right),
$$

where $\left(\mathbf{Z}^{n}, \leqslant_{\Gamma}\right)$ is the group of integers with the restricted preorder.

More explicitly, the isomorphism $\mathbf{Z}^{n} \cong H_{1}\left(\uparrow_{\Gamma} \mathbf{T}^{n}\right)$ in (53) restricts to a bijection between the positive cones of our preordered groups, $\Gamma^{\prime}\left(=\mathbf{Z}^{n} \cap \Gamma\right)$ and $\Gamma^{\prime \prime}$ (letting $p: \mathbf{R}^{n} \rightarrow \mathbf{R}^{n} / \mathbf{Z}^{n}$ denote the canonical projection)

$$
\begin{aligned}
\varphi: \Gamma^{\prime} \rightarrow \Gamma^{\prime \prime} \subset \uparrow H_{1}\left(\uparrow_{\Gamma} \mathbf{T}^{n}\right), & & \varphi(\rho)=\left[p a_{\rho}\right], \\
a_{\rho}: \mathbf{I} \rightarrow \mathbf{R}^{n} & & a_{\rho}(t)=t \rho .
\end{aligned}
$$

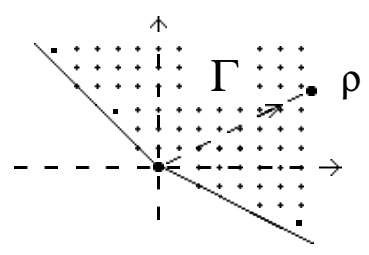


Proof. The hypothesis on $\Gamma$ ensures that the open subsets $x+\operatorname{int}(\Gamma)$ cover $\mathbf{R}^{n}$, and any two of them are contained in a third.

(A) The group $\mathbf{Z}^{n}$ acts properly (and pathwise freely) on $\mathbf{R}^{n}$, respecting $\leqslant_{\Gamma}$. Therefore $\mathbf{Z}^{n}$ acts (freely) on the cubical set $A=\square\left(\mathbf{R}^{n}, \leqslant_{\Gamma}\right)$, and we have (by (34))

$$
\uparrow H_{*}\left(\uparrow_{\Gamma} \mathbf{T}^{n}\right)=\uparrow H_{*}\left(\square\left(\mathbf{R}^{n}, \leqslant_{\Gamma}\right) / \mathbf{Z}^{n}\right)=\uparrow H_{*}\left(A / \mathbf{Z}^{n}\right) .
$$

(B) Now, we show that the cubical set $A$ is acyclic. Fixing some $x \in \mathbf{R}^{n}$, the preordered subspace $x+\Gamma \subset\left(\mathbf{R}^{n}, \leqslant_{\Gamma}\right)$ is contractible to its minimum $x$, by an obvious homotopy

$$
f:(x+\Gamma) \times \uparrow \mathbf{I} \rightarrow x+\Gamma, \quad f(y, t)=x+t(y-x) .
$$

Therefore, all the preordered subspaces $x+\Gamma$ are acyclic; but any cube of $\left(\mathbf{R}^{n}, \leqslant_{\Gamma}\right)$ has a compact image, contained in some $x+\Gamma$ (by the initial remark). It follows that also $\left(\mathbf{R}^{n}, \leqslant_{\Gamma}\right)$ is acyclic.

(C) Applying [15], Thm. 3.3, to the free action of $\mathbf{Z}^{n}$ on the acyclic cubical set $A$, the algebraic homology of the orbit cubical set is determined as in (53). But we want to show that this isomorphism is induced by the inclusion (52), which requires a finer analysis of the arguments on free actions of groups on cubical sets, developed in [15], Section 3.

Indeed, the augmented sequence

$$
\ldots \rightarrow C_{1}(A) \rightarrow C_{0}(A) \rightarrow \mathbf{Z} \rightarrow 0
$$

is exact, since $A$ is acyclic. By [15], 3.2a, this sequence is a $\mathbf{Z}^{n}$-free resolution of the trivial $\mathbf{Z}^{n}$-module $\mathbf{Z}$. Therefore, applying the isomorphism in [15], 3.2.1, and the definition of group-homology

$$
H_{k}\left(A / \mathbf{Z}^{n}\right) \cong H_{k}\left(C_{*}(A) \otimes \mathbf{Z}^{n} \mathbf{Z}\right) \cong H_{k}\left(\mathbf{Z}^{n}\right) .
$$

Similarly:

$$
H_{k}\left(\mathbf{R}^{n} / \mathbf{Z}^{n}\right) \cong H_{k}\left(C_{*}\left(\mathbf{R}^{n}\right) \otimes \mathbf{Z}^{n} \mathbf{Z}\right) \cong H_{k}\left(\mathbf{Z}^{n}\right),
$$

which shows that the embedding $A \subset \square \mathbf{R}^{n}$ (or $A / \mathbf{Z}^{n} \subset\left(\square \mathbf{R}^{n}\right) / \mathbf{Z}^{n}$, equivalently) induces an isomorphism in (algebraic) homology.

(D) Now, we want to determine the preorder of $\uparrow H_{1}$. We have two isomorphisms

$$
\lambda: H_{1}\left(\uparrow_{\Gamma} \mathbf{T}^{n}\right) \rightarrow H_{1}\left(\overline{\mathbf{T}}^{n}\right), \quad \mu: \mathbf{Z}^{n} \rightarrow H_{1}\left(\overline{\mathbf{T}}^{n}\right),
$$

the first is induced by the inclusion of directed cubes into arbitrary cubes, the second is computed as in (55), $\mu(\rho)=\left[p a_{\rho}\right]$ for $\rho \in \mathbf{Z}^{n}$. We shall use the isomorphism $\varphi=\lambda^{-1} \mu: \mathbf{Z}^{n} \rightarrow H_{1}\left(\uparrow_{\Gamma} \mathbf{T}^{n}\right)$.

Plainly, $\varphi$ restricts to an injection $\varphi: \Gamma^{\prime} \rightarrow \Gamma^{\prime \prime}$ as in (55): if $\rho \in \Gamma^{\prime}=\mathbf{Z}^{n} \cap \Gamma$, the path $a \rho(t)=t \rho$ is a directed 1 -cube of $\left(\mathbf{R}^{n}, \leqslant_{\Gamma}\right)$ and a (positive) cycle modulo $\mathbf{Z}^{n}$. We have to prove that $\varphi\left(\Gamma^{\prime}\right)$ covers $\Gamma^{\prime \prime}$ (the argument is similar to the one of $[\mathbf{1 5}]$, Thm. 4.8).

To simplify the argument, a 1-chain $z$ of $A$ which projects to a cycle $p_{*}(z)$ in $A / \mathbf{Z}^{n}$, or to a boundary, will be called a pre-cycle or a pre-boundary, respectively. (Note that, since $p_{*}$ is surjective, the homology of $A / \mathbf{Z}^{n}$ is isomorphic to the quotient 
of pre-cycles modulo pre-boundaries.) Let $z=\sum_{i} \lambda_{i} a_{i}$ be a positive pre-cycle, with all $\lambda_{i}>0$; let us call $\lambda=\sum_{i} \lambda_{i}$ its weight. We have to prove that $z$ is equivalent to a positive combination of pre-cycles of type $a_{\rho}\left(\rho \in \Gamma^{\prime}\right)$, modulo pre-boundaries.

Let $z=z^{\prime}+z^{\prime \prime}$, putting in $z^{\prime}$ all the summands $\lambda_{i} a_{i}$ which are pre-cycles themselves, and replace any such $a_{i}$, up to pre-boundaries, with $a_{\rho_{i}}$, where $\rho_{i}=$ $\partial^{1} a_{i}-\partial^{0} a_{i} \in \Gamma^{\prime}$. If $z^{\prime \prime}=0$ we are done, otherwise $z^{\prime \prime}=z-z^{\prime}$ is still a pre-cycle; let us act on it. Reorder its paths $a_{i}$ so that $a_{1}$ has a minimal coefficient $\lambda_{1}$ (strictly positive); since $\partial^{1} a_{1}$ has to annihilate in $\partial p_{*}\left(z^{\prime}\right)$, there is some $a_{i}(i>1)$ with $\partial^{1} a_{1}-\partial^{0} a_{i} \in \mathbf{Z}^{n}$. By a $\mathbf{Z}^{n}$-translation of $a_{i}$ (leaving $p a_{i}$ unaffected), we can assume that $\partial^{0} a_{i}=\partial^{1} a_{1}$, and then replace (modulo pre-boundaries) $\lambda_{1} a_{1}+\lambda_{i} a_{i}$ with $\lambda_{1} \hat{a}_{1}+\left(\lambda_{i}-\lambda_{1}\right) a_{i}$, where $\hat{a}_{1}=a_{1} * a_{i}$ is the concatenation (and $\lambda_{i}-\lambda_{1} \geqslant 0$ ). Now, the new weight is $\lambda-\lambda_{1}<\lambda$, strictly less than the previous one. Continuing this way, the procedure ends in a finite number of steps; this means that, modulo pre-boundaries, we have changed $z$ into a positive combination of pre-cycles of the required form, $a_{\rho}$.

\subsection{Comments}

Taking $n=2$, the previous result shows that the inequilogical spaces $\uparrow_{\Gamma} \mathbf{T}^{n}$ considered in 6.1 are really distinct, even up to local directed homotopy, since their preordered homology groups of degree 1

$$
\uparrow H_{1}\left(\uparrow_{\Gamma} \mathbf{T}^{n}\right)=\left(\mathbf{Z}^{2}, \leqslant_{\Gamma}\right),
$$

are not isomorphic. Indeed, taking $\Gamma=\Gamma_{1}, \ldots, \Gamma_{5}$, we have distinct results for $\left(\mathbf{Z}^{2}, \leqslant \Gamma\right)$ :

- $\Gamma_{1}$ : the lattice-ordered group $\uparrow \mathbf{Z} \times \uparrow \mathbf{Z}$; its positive cone has two atoms: $(1,0)$ and $(0,1)$

- $\Gamma_{2}$ : a lattice-ordered group; its positive cone has three atoms: $(1, y)$, with $y=$ $-1,0,1$,

- $\Gamma_{3}$ : a totally ordered, non-Archimedean group; its positive cone has one atom: $(0,1)$

- $\Gamma_{4}$ : an ordered group, not a lattice; the positive cone has countably many atoms: $(1, y)$, for $y \in Z$,

$-\Gamma_{5}$ : a preordered group.

Finally, every $\Gamma_{\vartheta}$ gives a totally ordered group, isomorphic to $\uparrow G_{\vartheta} \subset \uparrow \mathbf{R}$ and Archimedean

$$
\left(\mathbf{Z}^{2}, \leqslant_{\Gamma_{\vartheta}}\right) \rightarrow \uparrow G_{\vartheta}, \quad(x, y) \mapsto x+\vartheta y,
$$

whose isomorphism classes have been classified above (Thm. 4.4). It is easy to see that such classes correspond to the isomorphism classes of the inequilogical spaces $\left(\mathbf{R}^{2}, \leqslant_{\Gamma_{\vartheta}}, \equiv_{\mathbf{Z}^{2}}\right)$ : also here, it suffices to consider the cases $\zeta=\vartheta+k(k \in \mathbf{Z})$ and $\zeta=\vartheta^{-1}$, and the following isomorphisms of preordered topological spaces, consistent with the action of $\mathbf{Z}^{2}$

$$
\begin{array}{lll}
f:\left(\mathbf{R}^{2}, \leqslant \Gamma_{\zeta}\right) \rightarrow\left(\mathbf{R}^{2}, \leqslant \Gamma_{\vartheta}\right), & f(x, y)=(x+k y, y) & (\zeta=\vartheta+k) \\
f:\left(\mathbf{R}^{2}, \leqslant \Gamma_{\zeta}\right) \rightarrow\left(\mathbf{R}^{2}, \leqslant \Gamma_{\vartheta}\right), & f(x, y)=(y, x) & \left(\zeta=\vartheta^{-1}\right) .
\end{array}
$$


Finally, all these ordered groups $\left(\mathbf{Z}^{2}, \leqslant_{\Gamma_{\vartheta}}\right)$ are distinct from the previous ones, since the only total order previously obtained - the lexicographic one - is not Archimedean.

Total orders on the group $\mathbf{Z}^{n}$ (or on the additive monoids $\mathbf{N}^{n}$ ) are important for Gröbner bases and computer algebra. A description can be found in [26].

\section{References}

[1] A. Bauer - L. Birkedal - D.S. Scott, Equilogical spaces, Theoretical Computer Science, to appear.

[2] L. Birkedal - A. Carboni - G. Rosolini - D.S. Scott, Type theory via exact categories, in: Thirteenth Annual IEEE Symposium on Logic in Computer Science (Indianapolis, IN, 1998), 188-198, IEEE Computer Soc., Los Alamitos, CA, 1998.

[3] N. Bourbaki, Intégration, ch. 2, Hermann, Paris 1952.

[4] A. Carboni - E. Vitale, Regular and exact completions, J. Pure Appl. Algebra 125 (1998), 79-116.

[5] A. Connes, Noncommutative geometry, Academic Press, San Diego CA 1994.

[6] A. Connes, A short survey of noncommutative geometry, J. Math. Physics 41 (2000), 3832-3866.

[7] S. Eilenberg - S. Mac Lane, Acyclic models, Amer. J. Math. 75 (1953), 189-199.

[8] S. Eilenberg - N. Steenrod, Foundations of algebraic topology, Princeton Univ. Press 1952.

[9] L. Fajstrup - E. Goubault - M. Raussen, Algebraic topology and concurrency, Preprint R-99-2008, Dept. of Mathematical Sciences, Aalborg University, DK.

http://www .math.auc.dk/ fajstrup/publ.html

[10] P. Gaucher - E. Goubault, Topological deformation of higher dimensional automata, Homology, Homotopy Appl. 5 (2003), No. 2, 39-82.

http://www.rmi.acnet.ge/hha/volumes/2003/n2a3/

[11] E. Goubault, Geometry and concurrency: a user's guide, in: Geometry and concurrency, Math. Structures Comput. Sci. 10 (2000), no. 4, pp. 411-425.

[12] M. Grandis, Directed homotopy theory, I. The fundamental category, Cahiers Topologie Géom. Différentielle Catég. 44 (2003), 281-316.

[13] M. Grandis, Directed homotopy theory, II. Homotopy constructs, Theory Appl. Categ. 10 (2002), No. 14, 369-391.

http://tac.mta.ca/tac/

[14] M. Grandis, Ordinary and directed combinatorial homotopy, applied to image analysis and concurrency, Homology Homotopy Appl. 5 (2003), No. 
$2,211-231$.

http://www.rmi .acnet.ge/hha/volumes/2003/n2a7/

[15] M. Grandis, Directed combinatorial homology and noncommutative tori (The breaking of symmetries in algebraic topology), Math. Proc. Cambridge Philos. Soc., to appear. [Dip. Mat. Univ. Genova, Preprint 480 (2003).]

http://www.dima.unige.it/_grandis/Bsy.pdf

[16] M. Grandis, Normed combinatorial homology and noncommutative tori, Theory Appl. Categ., to appear [Dip. Mat. Univ. Genova, Preprint 484 (2003).]

http://www.dima.unige.it/ grandis/Bsy2.pdf

[17] M. Grandis, Equilogical spaces, homology and noncommutative geometry, Cah. Topol. Géom. Diff. Catég., to appear. [Dip. Mat. Univ. Genova, Preprint 493 (2003).]

http://www.dima.unige.it/_grandis/Eql.pdf

[18] P.J. Hilton - S. Wylie, Homology theory, Cambridge Univ. Press, Cambridge 1962.

[19] G.M. Kelly, Basic concepts of enriched category theory, Cambridge University Press, 1982.

[20] W.A.J. Luxemburg - A.C. Zaanen, Riesz spaces I, North-Holland, Amsterdam 1971.

[21] S. Mac Lane, Homology, Springer, Berlin 1963.

[22] W. Massey, Singular homology theory, Springer, Berlin 1980.

[23] M. Pimsner - D. Voiculescu, Imbedding the irrational rotation $\mathrm{C}^{*}$-algebra into an AF-algebra, J. Operator Th. 4 (1980), 93-118.

[24] M. Raussen, State spaces and dipaths up to dihomotopy, Homotopy Homology Appl. 5 (2003), No. 2, 257-280.

http://www.rmi.acnet.ge/hha/volumes/2003/n2a9/

[25] M.A. Rieffel, C*-algebras associated with irrational rotations, Pacific J. Math. 93 (1981), 415-429.

[26] L. Robbiano, Term orderings on the polynomial ring, EUROCAL '85, Vol. 2 (Linz, 1985), 513-517, Lecture Notes in Comput. Sci., 204, Springer, Berlin 1985.

[27] J. Rosický, Cartesian closed exact completions, J. Pure Appl. Algebra 142 (1999), 261-270.

[28] G. Rosolini, Equilogical spaces and filter spaces, Categorical studies in Italy (Perugia, 1997). Rend. Circ. Mat. Palermo (2) Suppl. No. 64, (2000), $157-175$. 
[29] D. Scott, A new category? Domains, spaces and equivalence relations, Unpublished manuscript (1996). http://www.cs.cmu .edu/Groups/LTC/

[30] A. Terras, Harmonic analysis on symmetric spaces and applications II, Springer, Berlin 1988.

This article may be accessed via WWW at http://www.rmi.acnet.ge/hha/ or by anonymous ftp at

ftp://ftp.rmi.acnet.ge/pub/hha/volumes/2004/n1a21/v6n1a21.(dvi,ps,pdf)

\author{
Marco Grandis \\ grandis@dima.unige.it \\ http://www.dima.unige.it/ grandis/ \\ Dipartimento di Matematica, \\ Università di Genova \\ Via Dodecaneso 35, 16146-Genova, Italy
}

